\title{
LOCALIZED WAVEFUNCTIONS AND MAGNETIC BAND STRUCTURE FOR LATERAL SEMICONDUCTOR SUPERLATTICES
}

\author{
G. S. Kliros ${ }^{a}$ and P.C. Divari ${ }^{b}$ \\ ${ }^{a}$ Department of Electronic and Communication Engineering \\ Hellenic Air-force Academy, Dekelia Air-Force Base, Attica TGA-1010, Greece \\ ${ }^{b}$ Department of Physics, University of Ioannina, GR-45110 Ioannina, Greece
}

February 4, 2020

\begin{abstract}
In this paper we present calculations on the electronic band structure of a two-dimensional lateral superlattice subject to a perpendicular magnetic field by employing a projection operator technique based on the ray-group of magnetotranslation operators. We construct a new basis of appropriately symmetrized Bloch-like wavefunctions as linear combination of well-localized magnetic-Wannier functions. The magnetic field was consistently included in the Wannier functions defined in terms of free-electron eigenfunctions in the presence of external magnetic field in the symmetric gauge. Using the above basis, we calculate the magnetic energy spectrum of electrons in a lateral superlattice with bi-directional weak electrostatic modulation. Both a square lattice and a triangular one are considered as special cases. Our approach based on group theory handles the cases of integer and rational magnetic fluxes in a uniform way and the provided basis could be convenient for further both analytic and numerical calculations.
\end{abstract}




\section{Introduction}

Lateral semiconductor superlattices are realized by modern lithographic techniques to impose a periodic pattern onto a two-dimensional electron system (2DES) at a semiconductor heterostructure. This pattern can be a uni- or bidirectional periodic potential modulation of different strength allowing for a variety of artificial periodic structures.[1, 2] Magnetotransport measurements in such structures have revealed novel oscillations in the conductivity, the Weiss oscillations which are superimposed on top of the well-known Shubnikov-de Haas oscillations. [3] Besides Weiss oscillations (a commensurability effect),novel magneto-resistance oscillations with $1 / B$-periodicity have been observed in short-period superlattices due to magnetic miniband energy spectrum. [4] The above developments have renewed the interest in the problem of Bloch electrons in a magnetic field, which was extensively studied in the past by renowned theorists[5] but it is experimentally accessible now with these artificial periodic structures.[6]

The first theoretical approach to the problem is due to Peierls[5] and it is based on the formulation of an effective single-band Hamiltonian, arising from a tight-binding dispersion relation $E(\boldsymbol{k})$, through the substitution $E(\boldsymbol{k}+e \boldsymbol{A} / \hbar)$, where $\boldsymbol{A}$ the vector potential. When combined to a semiclassical picture[7], the effective Hamiltonian theory leads to the idea of quantized magnetic orbits, which can be commensurate with the lattice period. Within the framework of effective single-band Hamiltonian theory, further investigations lead to butterflylike patterns for the energy spectrum known as Hofstadter spectrum. [8, 9] This spectrum reflects, as a consequence of Harper's equation[10], the splitting of the single Bloch-band into magnetic subbands, according to the number of magnetic flux quanta piercing the unit cell of the lattice. Some recent experimental results[11] supports the existence of the Hofstadter's spectrum in high magnetic field regime. Furthermore, an experimental realization of the Hofstadter's spectrum was achieved considering the transmission of microwaves[12] and acoustic waves[13] through an array of scatterers.

In recent years special attention has been paid to investigate the energy miniband structure[14] as well as the transport properties of two-dimensional short-period lateral supperlattices in the presence of a perpendicular magnetic field $\boldsymbol{B}$ in the case of weak modulation.[15, 16] Some calculations of superlattice electronic structure under the above conditions have used perturbation theory[17, 18] or a tight-binding scheme[19] and look for solutions of the oneelectron Hamiltonian that are linear combination of the unperturbed extended Landau wavefunctions. Another approach to the problem is based on Ferrari's wavefunctions[20], that is, wavefunctions constructed out of coherent states associated to a von Neumann-type lattice.[21] Besides the effect of electric modulation, the effect of magnetic modulation on the superlattice energy spectrum has been studied[22, 23] and a formal connection between these two has been investigated.[24]

In this paper we present calculations on the electronic band structure of a lateral superlattice subject to a perpendicular magnetic field by employing 
a projection operator technique in the context of magnetic translation group (M.T.G) theory. We use a new set of magnetic-consistent Wannier functions given in terms of Schrauben functions introduced by Jannussis[25] in order to construct Bloch-like eigenfunctions that reflect the crystal symmetry of the superlattice. Schrauben functions describe free electrons in a uniform magnetic field in the symmetric gauge and have been used in the past for magnetoconductance calculations. [26, 27]

The paper has the following structure: In Section 2 the model is formulated and the projection operator of the M.T.G corresponding to the problem is introduced. In Section 3 we construct a basis of appropriately symmetric Bloch-like wavefunctions as linear combination of Schrauben functions. In Section 4, the electronic spectrum of the 2DES, under weak periodic modulation, is obtained. In Section 5, we present specific results for periodic modulations with rectangular or triangular symmetry. The density of states is calculated as well. We conclude with a summarizing Section 6 .

\section{Projection operator of magnetic translation group}

To describe the electrons in the conduction band of a lateral superlattice at the AlGaAs-GaAs interface in a constant perpendicular magnetic field $\boldsymbol{B}=B \hat{z}$, we employ a model of strictly two-dimensional electron gas (2DEG) with a $2 \mathrm{D}$ periodic potential modulation $V(\boldsymbol{r}), \boldsymbol{r} \equiv(x, y)$.

Following the common practice, we adopt an effective mass approximation, to take into account the effect of the crystalline atomic structure over the charge carriers. The system is described by the well-known single-particle Hamiltonian of Bloch electrons in a magnetic field:

$$
\mathcal{H}=\frac{1}{2 m^{*}}(\boldsymbol{p}+e \boldsymbol{A})^{2}+V(\boldsymbol{r})
$$

where we neglect the Zeeman splitting and spin-orbit interactions which are very small for GaAs systems.

The Hamiltonian (1) does not commute with the usual translations operators

$$
\hat{T}(\boldsymbol{R})=\exp \left(-\frac{i}{\hbar} \boldsymbol{R} \cdot \boldsymbol{p}\right)
$$

where $\boldsymbol{R}$ is any translation vector, but commutes with unitary operators of the form

$$
\hat{T}_{m}(\boldsymbol{R})=\exp \left[-\frac{i}{\hbar} \boldsymbol{R} \cdot(\boldsymbol{p}-e \boldsymbol{A})\right]
$$

introduced by Brown[28] and Zak[29], if the vector potential $\boldsymbol{A}$ fulfills the condition

$$
\frac{\partial A_{j}}{\partial x_{k}}+\frac{\partial A_{k}}{\partial x_{j}}=0 \quad \text { for } \quad j, k=1,2,3
$$


This relation holds, for the symmetric gauge $\boldsymbol{A}(\boldsymbol{r})=\frac{1}{2}(\boldsymbol{B} \times \boldsymbol{r})$, which was used by both authors. It is worth noting that introducing local gauge one can consider any vector potential.[30, 31]

The above operators (3) form the magnetic translation group (M.T.G). This is in fact a ray group[32], because the product of two elements of the M.T.G yields another element, multiplied by a constant phase

$$
\hat{T}_{m}\left(\boldsymbol{R}_{1}\right) \hat{T}_{m}\left(\boldsymbol{R}_{2}\right)=\hat{T}_{m}\left(\boldsymbol{R}_{1}+\boldsymbol{R}_{2}\right) \exp \left[-\frac{i e}{\hbar} \boldsymbol{R}_{2} \cdot \boldsymbol{A}\left(\boldsymbol{R}_{1}\right)\right]
$$

where $\boldsymbol{R}_{1}$ and $\boldsymbol{R}_{2}$ are translation vectors. As a consequence of (5), we can verify the commutation relation

$$
\left[\hat{T}_{m}\left(\boldsymbol{R}_{1}\right), \hat{T}_{m}\left(\boldsymbol{R}_{2}\right)\right]=2 i \sin \left[-\frac{e}{2 \hbar} \boldsymbol{B} \cdot\left(\boldsymbol{R}_{1} \times \boldsymbol{R}_{2}\right)\right] \hat{T}_{m}\left(\boldsymbol{R}_{1}+\boldsymbol{R}_{2}\right)
$$

where the symmetric gauge for the vector potential has been used.

Therefore, the magnetic translation operators commute with the Hamiltonian, but in general not with each other. Only in the case that the phase constant in (6) is a multiple of $\pi$,

$$
\frac{e}{2 \hbar} \boldsymbol{B} \cdot\left(\boldsymbol{R}_{1} \times \boldsymbol{R}_{2}\right)=\pi q, \quad q \in \mathbb{N}
$$

we get $\left[\hat{T}_{m}\left(\boldsymbol{R}_{1}\right), \hat{T}_{m}\left(\boldsymbol{R}_{2}\right)\right]=0$ and the operators form an Abelian group. The condition (7) is compatible with the demand that the eigenfunctions of the system satisfy the Born-von Karman periodic boundary conditions, e.g. are periodic under magnetic translations corresponding to the full lattice size.

The term $\boldsymbol{B} \cdot\left(\boldsymbol{R}_{1} \times \boldsymbol{R}_{2}\right)$ in (7) is the magnetic flux through the cell spanned by lattice vectors $\boldsymbol{R}_{1}$ and $\boldsymbol{R}_{2}$ and can be written as an integer multiple $(p)$ of the flux per unit cell $\Phi=\Phi_{0} \boldsymbol{B} \cdot\left(\boldsymbol{a}_{1} \times \boldsymbol{a}_{2}\right)$, where $\Phi_{0}=e / \hbar$ is the magnetic flux quantum.

Consequently, the condition(7) is fulfilled each time the number of magnetic flux quanta through the unit cell is a rational number:

$$
\frac{\Phi}{\Phi_{0}}=\frac{q}{p}, \quad \text { with } \quad q, p \quad \text { mutually prime integers }
$$

thus, imposing a commensurability relation between the magnetic field intensity and the lattice size.

After a brief preamble on the M.T.G, we follow Brown's formulation[29] to introduce the projection operators of M.T.G. We consider the irreducible representations of the M.T.G labelled by the magnetic crystal momentum vector $\boldsymbol{q}$, restricted to a single magnetic Brillouin Zone (MBZ), or to $1 / p \times 1 / p$ part of it if $p \neq 1$. The $\boldsymbol{q}-t h$ irreducible representation is given by a familiar relation

$$
D^{\boldsymbol{q}}(\boldsymbol{R})=\exp (-i \boldsymbol{q} \cdot \boldsymbol{R}) D^{0}(\boldsymbol{R})
$$

where $\boldsymbol{R}=n_{1} \boldsymbol{a}_{1}+n_{2} \boldsymbol{a}_{2}$, with $\boldsymbol{a}_{1}, \boldsymbol{a}_{2}$ the primitive lattice vectors and $\boldsymbol{q}=$ $q_{1} \boldsymbol{b}_{1}+q_{2} \boldsymbol{b}_{2}$ with $\boldsymbol{b}_{1}, \boldsymbol{b}_{2}$ are the primitive reciprocal-lattice vectors. The vectors 
$\boldsymbol{a}_{1,2}$ and $\boldsymbol{b}_{1,2}$ are related in the usual fashion $\boldsymbol{a}_{i} \cdot \boldsymbol{b}_{j}=2 \pi \delta_{i j}$ and $n_{1,2}, q_{1,2}$ are integer indices.

The $p \times p$ matrix $D^{0}(\boldsymbol{R})$ for any translation $\boldsymbol{R}$ is generated from those corresponding to the primitive lattice vectors

$$
D_{k k^{\prime}}^{0}\left(\boldsymbol{a}_{1}\right)=\delta_{k k^{\prime}} \exp \left[2 \pi i(k-1) \frac{q}{p}\right], \quad D_{k k^{\prime}}^{0}\left(\boldsymbol{a}_{2}\right)=\delta_{k, k^{\prime}-1}^{\bmod p}
$$

and the ray-group multiplication law (5) yields to

$D_{k k^{\prime}}^{0}(\boldsymbol{R})=\delta_{k, k^{\prime}-n_{2}}^{\text {modp }} \exp \left[i \pi \frac{q}{p} n_{1}\left(n_{2}+2(k-1)\right)\right], \quad$ with $\quad k, k^{\prime}=0,1,2, \cdots p-1$

where the modulo Kronecker delta, $\delta_{i j}^{\bmod p}$ equals 1 if $i$ and $j$ are equal or differ by a multiple of $p$ and vanishes otherwise.

Using (9) and (11) we obtain the following form for the irreducible representations

$$
D_{k k^{\prime}}^{\left(q_{1}, q_{2}\right)}(\boldsymbol{R})=\delta_{k, k^{\prime}-n_{2}}^{\bmod p} \exp \left[-2 \pi i\left(n_{1} q_{1}+n_{2} q_{2}\right)\right] \exp \left[i \pi \frac{q}{p} n_{1}\left(n_{2}+2(k-1)\right)\right]
$$

We can see that increasing any of $q_{1}, q_{2}$ by $1 / p$ produces an equivalent representation. Thus the values of $\boldsymbol{q}$ have to be restricted to a single magnetic Brillouin Zone: $0 \leq q_{1}, q_{2}<1 / p$. The rank of matrices implies the existence of $p$-partner functions transforming according to different rows of the same representation $D_{k k^{\prime}}^{\left(q_{1}, q_{2}\right)}$

We introduce the projection operator of M.T.G. of Bloch electrons in a uniform magnetic field[34, 35]

$$
P_{k k^{\prime}}^{\boldsymbol{q}}(\boldsymbol{R})=\sum_{\boldsymbol{R}}\left[D_{k k^{\prime}}^{\boldsymbol{q}}(\boldsymbol{R})\right]^{*} \hat{T}_{m}(\boldsymbol{R})
$$

which projects out the component belonging to $k^{\prime}$-th row of the representation $\boldsymbol{q}$ and then generates the partner belonging to $k$-th row. Using the expression for the magnetic translation operator (3), Eq. (13) leads to

$$
P_{k k^{\prime}}^{\boldsymbol{q}}(\boldsymbol{R})=\sum_{\boldsymbol{R}} C_{\boldsymbol{R}} \exp (i \boldsymbol{q} \cdot \boldsymbol{R}) \exp \left[\frac{i}{\hbar} \boldsymbol{R} \cdot\left(\boldsymbol{p}-\frac{e}{2 \hbar} \boldsymbol{B} \times \boldsymbol{R}\right)\right]
$$

where

$$
C_{\boldsymbol{R}}=\delta_{k, k^{\prime}-n_{2}}^{\text {modp }} \exp \left[-i \pi \frac{q}{p} n_{1}\left(n_{2}+2(k-1)\right)\right]
$$

We finally remark that acting the projection operator $P^{q}(\boldsymbol{R})$ to an arbitrary function we can find a partner function of the $\boldsymbol{q}$-th representation of M.T.G. In the next section, we find a basis of Bloch electrons in a magnetic field by acting the found projection operator (14) onto a basis of free electrons in a magnetic field. 


\section{Localized wave-functions of Bloch electrons in a magnetic field}

Landau functions, eigenfunctions of angular momentum and localized functions based on coherent states[21, 36] are usually used to describe free electrons moving in a plane under the influence of a magnetic field. The coherent states[37] on a von Neumann lattice are the most localized eigenfunctions for an electron in magnetic field. For such a lattice, with a single flux quantum per unit cell, the area of the unit cell equals $2 \pi l^{2}$, where $l=(\hbar / e B)^{1 / 2}$ is the magnetic length.

Jannussis[25] working in the symmetric gauge, introduced a different basis of wave functions for free electrons in uniform magnetic field, who called them Schrauben functions:

$$
\psi_{n, \boldsymbol{k}}(\boldsymbol{r})=C_{n} \exp \left[-\left(\kappa_{x}^{2}+\kappa_{y}^{2}\right) l^{2}+i \boldsymbol{k} \cdot \boldsymbol{r}\right]\left(-\kappa_{y}-i \kappa_{x}\right)^{n}
$$

where $n \in \mathbb{N}$ is the Landau index, $\boldsymbol{k}$ the electron wavevector and $\kappa_{x}=k_{x}+y / 2 l^{2}$, $\kappa_{y}=k_{y}-x / 2 l^{2}$, The normalization coefficient is given by

$$
C_{n}=\sqrt{\frac{1}{2 \pi l^{2}} \frac{\left(2 l^{2}\right)^{n}}{n !}}
$$

The above functions have several important properties, some of which are:

i) They form a basis in the Hilbert space of free electrons in a uniform magnetic field.

$$
\begin{gathered}
\sum_{n, \boldsymbol{k}} \psi_{n, \boldsymbol{k}}^{*}(\boldsymbol{r}) \psi_{n, \boldsymbol{k}}\left(\boldsymbol{r}^{\prime}\right)=\delta\left(\boldsymbol{r}-\boldsymbol{r}^{\prime}\right) \\
\left(\psi_{n, \boldsymbol{k}}(\boldsymbol{r}), \psi_{n^{\prime}, \boldsymbol{k}}(\boldsymbol{r})\right)=\delta_{n n^{\prime}}
\end{gathered}
$$

ii)They are fully localized around the centers

$$
\boldsymbol{r}_{m}\left(x_{m}, y_{m}\right): x_{m}=l^{2} k_{y}, y_{m}=-l^{2} k_{x}
$$

and in the two-dimensional plane take the symmetric form :

$$
\begin{aligned}
\psi_{n, \boldsymbol{k}}(\boldsymbol{r})= & C_{n}\left[\left(x-x_{m}\right)^{2}-i\left(y-y_{m}\right)^{2}\right]^{n} \\
& \cdot \exp \left[-\frac{1}{4 l^{2}}\left[\left(x-x_{m}\right)^{2}+\left(y-y_{m}\right)^{2}+\frac{i}{2 l^{2}}\left(x_{m} y-x y_{m}\right)\right]\right]
\end{aligned}
$$

Also, the probability density $\rho=\psi^{*} \psi$ has a rotational symmetry about the centers $\left(x_{m}, y_{m}\right)$ and takes the form of the Poisson distribution

$$
\rho\left(r_{m}\right)=\frac{1}{2 \pi l^{2}} \frac{\left(r_{m}^{2} / 2 l^{2}\right)^{n}}{n !} \exp \left(-\frac{r_{m}^{2}}{2 l^{2}}\right)
$$

where $r_{m}^{2}=\left(x-x_{m}\right)^{2}+\left(y-y_{m}\right)^{2}$ 

$\boldsymbol{R}$

iii) They obey Harper's symmetric condition[10], i.e. for a lattice translation

$$
\psi_{n, \boldsymbol{k}}(\boldsymbol{r}+\boldsymbol{R})=\exp \left[\frac{i e}{\hbar} \boldsymbol{A}(\boldsymbol{R}) \cdot \boldsymbol{r}+i \boldsymbol{k} \cdot \boldsymbol{r}\right] \psi_{n, \boldsymbol{\kappa}}(\boldsymbol{r}) \quad, \quad \boldsymbol{\kappa}=\boldsymbol{k}-\frac{e}{\hbar} \boldsymbol{A}(\boldsymbol{R})
$$

It has been shown[38] that a Wannier function $w_{n}\left(\boldsymbol{r}-\boldsymbol{r}_{m}\right)$ for electrons in a magnetic field can be obtained directly form the Schrauben function if the wave-vector $\boldsymbol{k}$ is substituted by the vector potential $-e \boldsymbol{A}\left(\boldsymbol{r}_{m}\right) / \hbar$

In what follows, we construct a basis for two-dimensional Bloch electrons in a uniform magnetic field applying the projection operator method described in Section 2. In order to find the principal partner function we choose the projection operator $P_{11}^{q}(\boldsymbol{R})$ (see Eq. (14)), projecting onto the first row of $\boldsymbol{q}$-th representation.

Acting the projection operator Eq. (14) onto the functions $\psi_{n, 0}(\boldsymbol{r})$ of the basis (16) we find the following partner functions of the $\boldsymbol{q}$-th representation of the M.T.G.

$$
\begin{aligned}
\Psi_{n, \boldsymbol{q}}(\boldsymbol{r}) & =\sum_{\boldsymbol{R}} C_{\boldsymbol{R}} \exp (i \boldsymbol{q} \cdot \boldsymbol{R}) \psi_{n, \boldsymbol{k}_{0}}(\boldsymbol{r}) \\
& =\sum_{\boldsymbol{R}} C_{\boldsymbol{R}} \exp (i \boldsymbol{q} \cdot \boldsymbol{R}) w_{n}(\boldsymbol{r}-\boldsymbol{R}), \quad \boldsymbol{k}_{0}=-\frac{e}{\hbar} \boldsymbol{A}(\boldsymbol{R})
\end{aligned}
$$

where $w_{n}(\boldsymbol{r}-\boldsymbol{R})$ are the magnetic Wannier functions of free electrons in a uniform magnetic field[38],

$$
\begin{aligned}
w_{n}(\boldsymbol{r}-\boldsymbol{R})= & C_{n}\left\{\frac{1}{2 l^{2}}\left[\left(x-n_{1} a_{1}\right)+i\left(y-n_{2} a_{2}\right)\right]\right\}^{n} \\
& \cdot \exp \left\{-\frac{1}{4 l^{2}}\left[\left(x-n_{1} a_{1}\right)^{2}+\left(y-n_{2} a_{2}\right)^{2}\right]+\frac{i}{2 l^{2}}\left(y n_{1} a_{1}-x n_{2} a_{2}\right)\right\}
\end{aligned}
$$

where $a_{1}, a_{2}$ are the 2D-lattice periods along $x$ and $y$ directions respectively and the normalization constant $C_{n}$ is given by Eq.(17).

Therefore, Eq. (24) represents modified Bloch functions which are formally expanded in the corresponding magnetic Wannier functions, that is, functions strongly localized on the lattice sites. Note that, for commensurate magnetic fields (7) one can recover Bloch's theorem

$$
\hat{T}(\boldsymbol{a}) \Psi_{n, \boldsymbol{q}}(\boldsymbol{r})=\exp (-i \boldsymbol{q} \cdot \boldsymbol{a}) \Psi_{n, \boldsymbol{q}}(\boldsymbol{r})
$$

with the values of the magnetic crystal momentum $\boldsymbol{q}=\left(q_{x}, q_{y}\right)$ restricted to the first magnetic Brillouin zone. Also, $\Psi_{n, \boldsymbol{q}}$ with different values of $\boldsymbol{q}$ are orthogonal since they belong to different irreducible representations of M.T.G. Finally, after some straightforward steps, we arrive at the following expression 
for the eigenfunctions Eq. (24)

$$
\begin{aligned}
\Psi_{n, \boldsymbol{q}}(\boldsymbol{r})= & \sum_{n_{1} n_{2}} C_{n_{1} n_{2}} C_{n} \exp \left[i\left(q_{x} n_{1} a_{1}+q_{y} n_{2} a_{2}\right)\right]\left\{\frac{1}{2 l^{2}}\left[\left(x-n_{1} a_{1}\right)+i\left(y-n_{2} a_{2}\right)\right]\right\}^{n} \\
& \cdot \exp \left\{-\frac{1}{4 l^{2}}\left[\left(x-n_{1} a_{1}\right)^{2}+\left(y-n_{2} a_{2}\right)^{2}\right]+\frac{i}{2 l^{2}}\left(y n_{1} a_{1}-x n_{2} a_{2}\right)\right\}
\end{aligned}
$$

where $C_{n_{1} n_{2}}=\exp \left(-i \pi n_{1} n_{2} q / p\right)$ with $n_{2}$ integer multiple of $p$. The values of crystal momentum $\boldsymbol{q}=\left(q_{x}, q_{y}\right)$ are determined from the periodic boundary conditions for $\Psi_{n, \boldsymbol{q}}(\boldsymbol{r})$ with respect to magnetic translations with periods $a_{1}$ and $a_{2}$.

Figure 1 shows the contour plots of the modulus $\left|\Psi_{0, \boldsymbol{q}}(\boldsymbol{r})\right|$ for the case of a square periodic lattice $\left(a_{1}=a_{2}=100 \mathrm{~nm}\right)$ in the presence of a perpendicular magnetic field that corresponds to two flux quanta per unit cell, for different values of the crystal momentum $\boldsymbol{q}$ in the first Brillouin zone. It is seen that the wavefunctions possess the full symmetry of the lattice and have regularly distributed zeros as it is expected from the translational invariance of the system.

\section{Electron energy spectrum under weak peri- odic modulation potential}

Now we can find the energy spectrum of Bloch electrons in a constant magnetic field and weak periodic modulation potential $V(\boldsymbol{r})=V(\boldsymbol{r}+\boldsymbol{R})$ using the basis $\{|n, \boldsymbol{q}\rangle\}$ introduced in the preceding Section 3 .

It is well-known[25] that the matrix elements of the first part of the Hamiltonian (1) which describes free electrons in a uniform magnetic field are given by

$$
\left\langle n, \boldsymbol{q}\left|\frac{1}{2 m^{*}}(\boldsymbol{p}+e \boldsymbol{A})^{2}\right| n^{\prime}, \boldsymbol{q}\right\rangle=\left(n+\frac{1}{2}\right) \hbar \omega_{c} \delta_{n n^{\prime}}
$$

where $\omega_{c}=e B / m^{*}$ is the cyclotron frequency and $n$ labels the Landau levels.

In order to find the corresponding energy spectrum of Bloch electrons we first evaluate the matrix elements of the periodic potential. Since $V(\boldsymbol{r})$ is periodic with respect to $\boldsymbol{R}$ it can be expressed in terms of a Fourier series

$$
V(\boldsymbol{r})=\sum_{\boldsymbol{G}} V_{\boldsymbol{G}} \exp (i \boldsymbol{G} \cdot \boldsymbol{r})
$$

with the Fourier coefficients given by

$$
V_{\boldsymbol{G}}=\frac{1}{A} \int_{A} d^{2} r V(\boldsymbol{r}) \exp (-i \boldsymbol{G} \cdot \boldsymbol{r})
$$

where $A$ stands for the area of the unit cell in the two-dimensional lattice and $\boldsymbol{G}$ is a reciprocal lattice vector. 
In the two dimensional lattice $(x, y)$ the potential of Eq. (29) is written as

$$
V(x, y)=\sum_{g_{1} g_{2}} V_{g_{1}, g_{2}} \exp \left[i\left(\frac{2 \pi}{a_{1}} g_{1} x+\frac{2 \pi}{a_{2}} g_{2} y\right)\right]
$$

with $g_{1}, g_{2}$ integers and $a_{1}, a_{2}$ the modulation periods in $x$ and $y$ direction, respectively.

Using the basis (27), the matrix elements of the potential $V(x, y)$ can be derived for each crystal momentum $\boldsymbol{q}=\left(q_{x}, q_{y}\right)$ :

$$
\begin{aligned}
V_{n n^{\prime}} \equiv & \left\langle n, \boldsymbol{q}|V(x, y)| n^{\prime}, \boldsymbol{q}\right\rangle=\sum_{g_{1} g_{2}} \sum_{n_{1} n_{2}} \sum_{n_{1}^{\prime} n_{2}^{\prime}} C_{n_{1} n_{2}}^{*} C_{n_{1}^{\prime} n_{2}^{\prime}} V_{g_{1} g_{2}} \\
& \cdot \exp \left[i q_{x} a_{1}\left(n_{1}-n_{1}^{\prime}\right)+i q_{y} a_{2}\left(n_{2}-n_{2}^{\prime}\right)\right] \\
& \cdot \exp \left[-\frac{a_{2}^{2}}{l^{2}}\left(n_{2}-n_{2}^{\prime}\right)\left(n_{2}-n_{2}^{\prime}+2 g_{1} \frac{\Phi_{0}}{\Phi}\right)-\frac{a_{1}^{2}}{l^{2}}\left(n_{1}-n_{1}^{\prime}\right)\left(n_{1}-n_{1}^{\prime}-2 g_{2} \frac{\Phi_{0}}{\Phi}\right)\right] \\
& \cdot \exp \left[i \pi\left[\left(n_{1}^{\prime} n_{2}-n_{2}^{\prime} n_{1}\right) \frac{\Phi}{\Phi_{0}}+\left(n_{1}+n_{1}^{\prime}\right) g_{1}+\left(n_{2}+n_{2}^{\prime}\right) g_{2}\right]\right] I_{n n^{\prime}}\left(g_{1}, g_{2}\right)
\end{aligned}
$$

where

$$
\begin{aligned}
I_{n n^{\prime}}\left(g_{1}, g_{2}\right)= & (-1)^{n+n^{\prime}} \sqrt{\frac{n^{\prime} !}{n !}}\left(\sqrt{\frac{a_{1}}{a_{2}}} g_{2}+i \sqrt{\frac{a_{2}}{a_{1}}} g_{1}\right)^{n-n^{\prime}}\left(\pi \frac{\Phi_{0}}{\Phi}\right)^{\left(n-n^{\prime}\right) / 2} \\
& \cdot \exp (-u / 2) L_{n^{\prime}}^{n-n^{\prime}}(u), \quad \text { for } \quad n \geq n^{\prime}
\end{aligned}
$$

and

$$
\begin{aligned}
I_{n n^{\prime}}\left(g_{1}, g_{2}\right)= & (-1)^{n+n^{\prime}} \sqrt{\frac{n !}{n^{\prime} !}}\left(-\sqrt{\frac{a_{1}}{a_{2}}} g_{2}+i \sqrt{\frac{a_{2}}{a_{1}}} g_{1}\right)^{n^{\prime}-n}\left(\pi \frac{\Phi_{0}}{\Phi}\right)^{\left(n^{\prime}-n\right) / 2} \\
& \cdot \exp (-u / 2) L_{n}^{n^{\prime}-n}(u), \quad n^{\prime} \geq n
\end{aligned}
$$

with $u=\pi\left(\frac{a_{2}}{a_{1}} g_{1}^{2}+\frac{a_{1}}{a_{2}} g_{2}^{2}\right) \frac{\Phi_{0}}{\Phi}$ and $L_{m}^{n}(u)$ the associate Laguerre polynomials.

Furthermore, from the expression (32) one sees that for high magnetic fields or if the lattice points are far apart each other, the matrix elements $V_{n n^{\prime}}$ approach zero unless the following conditions are valid:

$$
n_{1}-n_{1}^{\prime}=2 g_{2} \frac{\Phi_{0}}{\Phi} \quad \text { and } \quad n_{2}-n_{2}^{\prime}=-2 g_{1} \frac{\Phi_{0}}{\Phi}
$$

Under the above conditions, which mean that the magnetic flux through a unit cell of the periodic lattice should be a rational number, the matrix elements (32) take the form

$$
V_{n n^{\prime}}=\sum_{g_{1} g_{2}} V_{g_{1} g_{2}} \exp \left[2 i \frac{\Phi_{0}}{\Phi}\left(g_{2} a_{1} q_{x}-g_{1} a_{2} q_{y}\right)\right] I_{n n^{\prime}}\left(g_{1}, g_{2}\right)
$$


Finally, the first order energy perturbation can be found by calculating the diagonal matrix elements from Eqs. (36) and (33):

$$
V_{n n}\left(q_{x}, q_{y}\right)=\sum_{g_{1} g_{2}} V_{g_{1} g_{2}} \exp \left[2 i \frac{\Phi_{0}}{\Phi}\left(g_{2} a_{1} q_{x}-g_{1} a_{2} q_{y}\right)\right] \exp (-u / 2) L_{n}(u)
$$

Thus, the complete energy spectrum to first order approximation can be written as

$$
E_{n}\left(q_{x}, q_{y}\right)=\left(n+\frac{1}{2}\right) \hbar \omega_{c}+V_{n n}\left(q_{x}, q_{y}\right)
$$

\section{Energy band structure for lateral superlat- tices with weak modulation}

In this section, we present analytical results concerning the energy spectrum of superlattices with perfect rectangular or triangular symmetry applying the formalism of the preceding Section 4. We consider smooth periodic potentials described by a few lowest Fourier components with weak modulation strengths.

It is well-known that the numerical solution of the corresponding tightbinding equation[39] for 2D-lattices in perpendicular magnetic field, shows that each Landau level splits into several sub-bands when a rational number of flux quanta $\Phi_{0}$ pierces the unit cell and that the corresponding gaps are exponentially small. In what follows, we assume that the gaps are closed due to disorder in samples of not exceptionally high mobilities, that is, we assume that the Landau levels are bands neglecting the above fine structure. This assumption is justified since the reported experiments[40]did not indicate that this fine structure of the energy spectrum was resolved.

\subsection{Lateral superlattices with rectangular symmetry}

We consider a two-dimensional periodic modulation with rectangular symmetry, i.e:

$$
V(x, y)=V_{x} \cos \left(\frac{2 \pi}{a_{1}} x\right)+V_{y} \cos \left(\frac{2 \pi}{a_{2}} y\right)
$$

where $V_{x}, V_{y}$ are the modulation strengths and $a_{1} a_{2}$ the periods in the corresponding directions. We assume that the modulation is weak compared to the cyclotron energy $\hbar \omega_{c}$ so that Eq. (38) is valid and mixing of different Landau levels can be neglected.

In terms of the plane wave expansion Eq. (31), the periodic potential (39) can be described by four Fourier coefficients

$$
V_{( \pm 1,0)}=V_{x} / 2 \quad \text { and } \quad V_{(0, \pm 1)}=V_{y} / 2
$$

Applying Eq. (38) we find the energy spectrum

$$
E\left(q_{x}, q_{y}\right)=\left(n+\frac{1}{2}\right) \hbar \omega_{c}+V_{x} F\left(u_{x}\right) \cos \left(\frac{2 a_{1}}{\pi} u_{x} q_{y}\right)+V_{y} F\left(u_{y}\right) \cos \left(\frac{2 a_{2}}{\pi} u_{y} q_{x}\right)
$$


where

$$
F(u)=\exp (-u / 2) L_{n}(u), \quad u_{x}=\frac{a_{2}}{a_{1}}\left(\pi \Phi_{0} / \Phi\right) \quad \text { and } \quad u_{y}=\frac{a_{1}}{a_{2}}\left(\pi \Phi_{0} / \Phi\right)
$$

Thus, the unperturbed Landau levels broaden into bands, with width equal to $2\left(V_{x}\left|F_{n}\left(u_{x}\right)\right|+V_{y}\left|F_{n}\left(u_{y}\right)\right|\right)$, that oscillates with inverse magnetic flux $\Phi_{0} / \Phi$ . Using the asymptotic form of Laguerre polynomials in the large n-limit, it can be shown that the bandwidth tends to zero when $2 \sqrt{n \pi}=(\lambda+1 / 4) \pi$ and reaches maximum when $2 \sqrt{n \pi}=(\lambda-1 / 4) \pi$ with $\lambda$ an integer. Therefore, the energy bandwidths in Eq. (40) oscillate with the Landau index. One important consequence of the non-zero bandwidth is finite electron-velocities leading to a finite band conductivity[41] which is absent when the modulation is not present.

In Fig.2 we plot the energy spectrum for the first two Landau levels, $n=0,1$, modulation strengths $V_{x}=V_{y}=0.5 \mathrm{meV}$ and for (a) an integer $\left(\Phi / \Phi_{0}=2\right)$ and (b) a rational $\left(\Phi / \Phi_{0}=5 / 4\right)$ number of flux quanta. The square superlattice period has been taken $a=100 \mathrm{~nm}$. It is seen that the energy spectrum reflects the symmetry of modulated square lattice.

The interesting characteristics in the energy spectrum of the weak modulated superlattice are also reflected in the density of states (DOS) per unit area defined by

$$
g(E)=\frac{g_{s}}{2 \pi l^{2}} \sum_{n=0}^{\infty} \sum_{q_{x} q_{y}} \delta\left[E-E_{n}\left(q_{x}, q_{y}\right)\right]
$$

where $g_{s}=2$ is the electro-spin degeneracy. For the spectrum given by Eq. (40) the DOS per unit area takes the following form:

$g(E)=g_{0} \sum_{n=0}^{\infty} \int_{0}^{2 \pi} d \theta\left\{\left[V_{x} F_{n}\left(u_{x}\right)\right]^{2}-\left[E-(n+1 / 2) \hbar \omega_{c}-V_{y} F_{n}\left(u_{y}\right) \cos \theta\right]^{2}\right\}^{-1 / 2}$

where $g_{0}=1 /\left(\pi^{3} l^{2}\right)$. Figure 3 plots the DOS per unit area for the same values of parameters as in Fig.2. As can be seen, the DOS does not exhibit Van Hove singularities at the edges of each Landau band reflecting the 2D nature of the electron motion in this band with finite mean velocities. Finally, we have to note that similar results for the band structure of a two dimensional surface superlattice have been obtained previously by direct application of perturbation theory to a different unperturbed basis of extended Landau wavefunctions.[18, $39]$

\subsection{Lateral superlattices with triangular symmetry}

We now consider a laterally modulated 2DEG with triangular symmetry, i.e:

$$
V(x, y)=V_{x} \cos \left(\frac{2 \pi}{a_{1}} x\right) \cos \left(\frac{2 \pi}{a_{2}} y\right)+V_{y} \cos \left(\frac{4 \pi}{a_{2}} y\right)
$$


where $a_{1}=a$ and $a_{2}=\sqrt{3} a$. This periodic modulation can be realized in a GaAs/AlGaAs heterostructure by means of a diblock copolymer nanolithography technique.[42]

The above periodic potential can be described by six Fourier coefficients

$$
V_{(1, \pm 1)}=V_{(-1, \pm 1)}=V_{x} / 4 \quad \text { and } \quad V_{(0, \pm 2)}=V_{y} / 2
$$

where we assume again the condition of weak modulation $V_{x}, V_{y}<<\hbar \omega_{c}$. Applying Eq. (38) we find the energy spectrum

$$
\begin{aligned}
E\left(q_{x}, q_{y}\right) & =(n+1 / 2) \hbar \omega_{c}+V_{x} F\left(u_{x}+u_{y}\right) \cos \left(\frac{2 a}{\pi} u_{x} q_{y}\right) \cos \left(\frac{2 \sqrt{3} a}{\pi} u_{y} q_{x}\right) \\
& +V_{y} F\left(4 u_{y}\right) \cos \left(\frac{4 \sqrt{3} a}{\pi} u_{y} q_{x}\right)
\end{aligned}
$$

where the parameters $u_{x}, u_{y}$ and the function $F_{n}(u)$ are defined in the preceding subsection 5.1.

As in the rectangular case, the Landau levels have broadened into bands with a bandwidth equal to $2\left(V_{x}+V_{y}\right) \mid F_{n}\left(4 u_{y}\right)$, that oscillates with inverse magnetic flux $\Phi_{0} / \Phi$ and Landau band index n. In Fig.4 the energy bands for $n=0$ and $n=1$, are plotted for the same values of parameters as in the rectangular case. The energy spectrum reflects the symmetry of the modulated triangular lattice.

Furthermore, the DOS per unit area of the superlattice with triangular symmetry can be calculated according to Eq. (41) and is given by

$$
\begin{aligned}
g(E)= & g_{0} \sum_{n=0}^{\infty} \int_{0}^{2 \pi} d \theta\left\{\left[V_{x} F_{n}\left(u_{x}+u_{y}\right) \cos \theta\right]^{2}\right. \\
& \left.-\left[E-(n+1 / 2) \hbar \omega_{c}-V_{y} F_{n}\left(4 u_{y}\right) \cos 2 \theta\right]^{2}\right\}^{-1 / 2}
\end{aligned}
$$

where $g_{0}=1 /\left(\pi^{3} l^{2}\right)$. The DOS for this case has similar characteristics to the one shown in Fig. 3 and is not plotted.

\subsection{Exact energy spectrum}

The approximate analytic energy spectrum of the preceding subsections 5.1 and 5.2 , provides a good insight into the problem and can facilitate the study of other properties, e.g., the thermodynamic and the transport properties of the superlattice. On the other hand, the exact energy spectrum can be obtained by diagonalizing numerically the Hamiltonian matrix produced by means of Eq.(36). The diagonalization is carried out by truncating the matrix dimensions while including a sufficient number of Landau levels. We were careful to choose the rank of the matrix being diagonalized large enough to ensure the convergence of our result. In Fig.5 the exact energy spectrum of the superlattice with square symmetry, given by Eq.(40) and the approximate one are plotted as a function of the crystal momentum $\left(q_{x}, q_{y}=0\right)$ for the first ten Landau levels, where the modulation strengths are $V_{x}=V_{y}=0.5 \mathrm{meV}$ and $\Phi / \Phi_{0}=2$. 
We see that the approximate energy spectrum (solid curve) agrees well in the whole range of crystal momentum with the exact one (dotted line). Similar agreement is found for other values of magnetic flux $\Phi / \Phi_{0}$ provided that the condition of weak modulation is satisfied.

\section{Concluding Remarks}

In summary, we have studied the energy spectrum of electrons in lateral semiconductor superlattices subject to a perpendicular magnetic field. Such systems, in which the simultaneous influence of a bi-directional periodic potential and a magnetic field is studied, are realized nowadays in layered semiconductor structures with modern technologies of growth and lithography.

We introduced a projection operator method applied to irreducible representations of the magnetotranslation group in order to construct an appropriately symmetrized basis of Bloch-type wavefunctions in terms of a new set of magnetic-consistent Wannier functions. The well-localized Wannier functions in this linear combination, were produced by eigenfunctions of free electrons in a magnetic field obtained in the symmetric gauge.

Using the above Bloch-type wavefunctions we have calculated the magnetic energy spectrum of Bloch-electrons in a two dimensional periodic potential obtaining similar results to previous pertubative or numerical approaches. The spectrum is expressed as a function of crystal-momenta, defined in a magnetic Brillouin zone, which are good quantum numbers of the system. Especially, we have presented explicit results concerning the band structure of short-period lateral superlattices with both square and triangular symmetry. The obtained oscillatory behavior of energy bandwidths, in the weak modulation regime, manifest themselves in the magnetoresistance measurements where they give rise to the so called Weiss oscillations in the dependance of the conductance on the magnetic field strength.

Finally, we note that our approach provides a symmetry-adapted basisfunctions based on group theory and handles the cases of integer and rational magnetic flux in a uniform way. For rational magnetic fluxes it is assumed that the fine structure of Landau bands can be neglected in samples of not exceptionally high mobilities, since the mini-gaps in a Landau band are closed due to disorder. From the presented results, it seems that our basis is appropriate in the intermediate regime of magnetic fields where both the lattice period and the magnetic length are comparable in magnitude. We also expect that this basis is convenient for further both analytic and numerical calculations. 


\section{References}

[1] F. M. Peeters and J. D. Boeck, Handbook of Nanostructured Materials and Nanotechnology Vol. 3, ed. H. S. Nalwa (New York, Academic Press, 2000) ch. 7 .

[2] D. Weiss, Ad. Solid State Phys. 31341 (1991); R. W. Winkler, J. P. Kotthaus and K. Ploog Phys. Rev. Lett. 621177 (1989).

[3] R. A. Deutschmann et al.,Phys. Rev. Lett. 861857 (2001).

[4] C. Albrecht et al.,Physica B 249-251 914 (1998).

[5] P. Peierls, Z. Phys. 80763 (1933); J. M. Luttinger, Phys. Rev. 133 A 1030 (1956); G. H. Wannier,Rev. Mod. Phys. 34645 (1962); E. Brown, Phys. Rev. A166 626 (1968); J. Zak, Phys. Rev. 1771151 (1969).

[6] C. Albrecht et al.,Phys. Rev. Lett. 832234 (1999).

[7] L. Onsager, Philos. Mag. 431006 (1952); A. B. Pippard, Proc. R. Soc. London SerA 256317 (1964); W. G. Chambers, Phys. Rev. 140 A135 (1965);

[8] F. H. Fofstadter, Phys. Rev. B 142239 (1976).

[9] R. A. Claro and G. H. Wannier Phys. Rev. B 196068 (1979).

[10] P.G. Harper, Proc. Phys. Soc. London A68 874 (1955).

[11] C. Albrecht et al., Phys. Rev. Lett. 86147 (2001); C. Albrecht et al., Physica E 20143 (2003).

[12] U. Kuhl and H. Stockmann, Phys. Rev. Lett. 803232 (1998).

[13] D. Richoux and V. Pagneux, Europhys. Lett. 5934 (2002).

[14] D. E. Grant, A. R. Long and J. H. Davies Phys. Rev. B 6113127 (2000).

[15] U. Rossler and M. Suhrke, Ad. Solid State Phys. 4035 (2000).

[16] M. Langenbuch, M. Suhrke and U. Rossler Europhys. Lett. 61520 (2003).

[17] N. Usov,Zh. Eksp. Teor. Fiz. 94305 (1988) [Sov. Phys. Jetp 672564 (1988)]

[18] F. M. Peeters and P. Vasilopoulos Phys. Rev. B42 5899 (1990); Phys. Rev. B46 4667 (1992).

[19] I. Labbe Phys. Rev. B35 1373 (1987);

[20] H. Silberbauer, J. Phys. : Contens. Matter 47355 (1992); V. Gudmundsson and R. R. Gerhards, Phys. Rev. B52,16744-16752 (1995). 
[21] R. Ferrari, Phys. Rev. B42 4598 (1990); R. Ferrari, Int. J. Mod. Phys. 9 3333 (1995).

[22] Q. W. Shi and K. Y. Szelto, Phys. Rev. B56 9251 (1997); G. Y. Oh, Phys. Rev. B60 1939 (1999);

[23] M. C. Chang and M. F. Yang, Phys. Rev. B69 115108 (2004).

[24] M. C. Chang and Q. Niu, Phys. Rev. B50 10843 (1994).

[25] A. Jannussis, Phys. Stat. Sol. 6217 (1964).

[26] K. Chung and B. Mrowka, Z. Physik 259 (1973).

[27] G. S. Kliros, E. Skuras and A. Jannussis, Nuovo Cimento D 12879 (1990).

[28] E. Brown, Phys. Rev. 1021038 (1964).

[29] J. Zak, Phys. Rev. 134 A 1602 (1964).

[30] P. Wiegmann and A. Zabrodin, Phys. Rev. Lett. 721890 (1994).

[31] W. Florek, J.Phys: Condens Matter 112523 (1999);

[32] E. Brown, Aspects of Group Theory in Electronic Dynamics, Solid State Physics, Vol. 22, (Academic Press, N. York and London, 1969).

[33] M. Hamermesh,Group Theory and its Applications to Physical Problems, (Dover, New York, 1989) chap. 12

[34] S. L. Altmann,Induced Representations in Crystals and Molecules , (London, Academic Press, 1977).

[35] H. J. Fischbeck, Phys. Stat. Sol. 3811 (1970).

[36] D. A. Hutchinson, J. C. Inkson and J. M. Rowe, Solid State Commun. 97 515 (1996).

[37] A. Feldman and A. H. Kahn, Phys. Rev. B1 4584 (1970); I. A. Malkin and V. I. Manko, Sov. Phys. JETP 28527 (1969).

[38] A. Jannussis,Z. Naturforsch. 21a 1577 (1961); G. Gountaroulis, Phys. Lett. A40 132 (1972)

[39] X. F. Wang, P. Vasilopoulos and F. M. Peeters, Phys. Rev. B69 035331 (2004).

[40] S. Chowdhury, A. Long, J. Davies, K. Lister and E. Skuras, Proceedings of EP2DS-14, p.583 (2001); S. Chowdhury, A. Long, E. Skuras, J. Davies, K. Lister, G. Panneli and C. Stanley, Phys. Rev. B69 035330 (2004).

[41] P. Vasilopoulos and F. M. Peeters, Phys. Rev. Lett. 632120 (1989).

[42] S. Melinte et al., Phys. Rev. Lett. 92036802 (2004). 

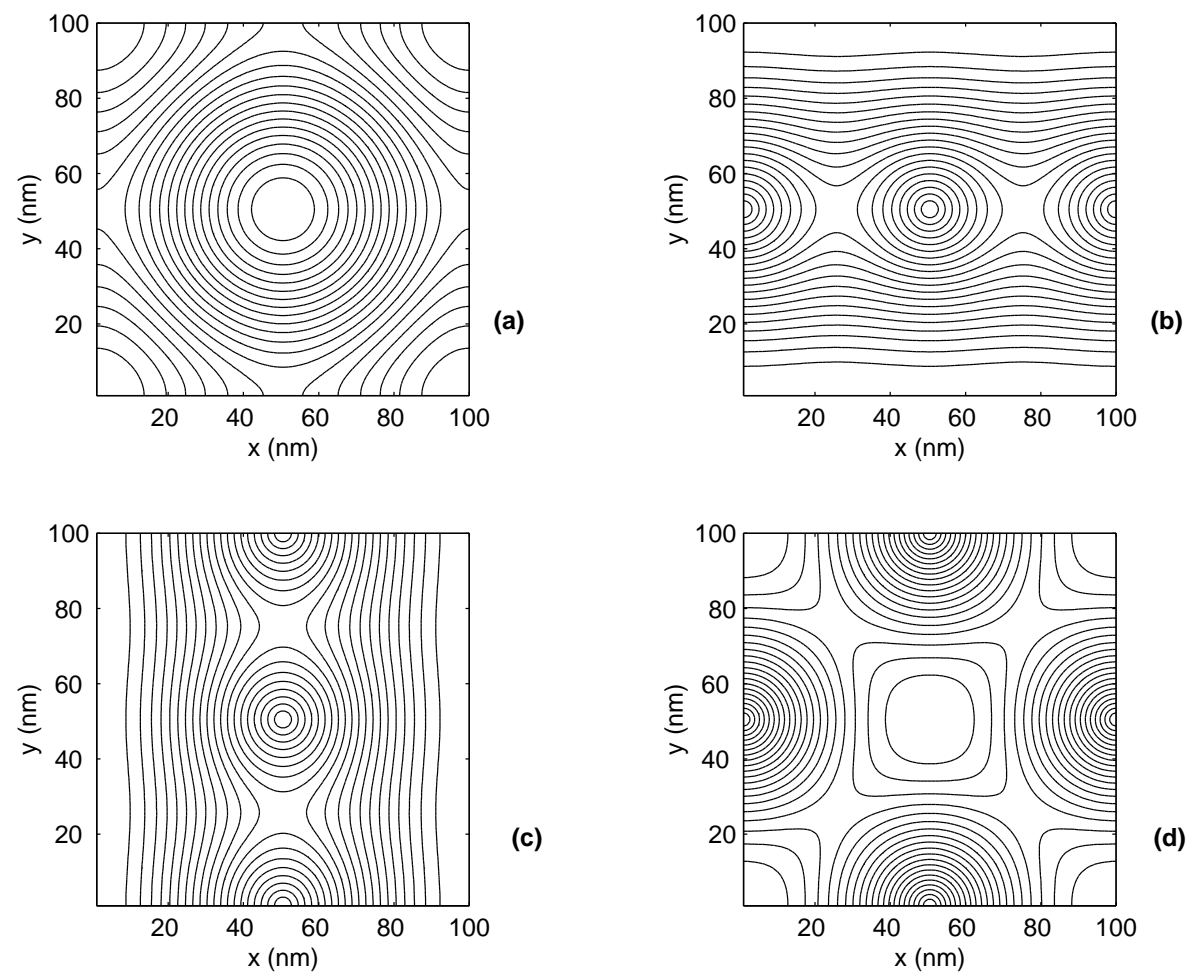

Figure 1: Contour plots of the modulus $\left|\Psi_{0, \boldsymbol{q}}(\boldsymbol{r})\right|$ for a square lattice with $a=$ $100 \mathrm{~nm}$ and $\Phi / \Phi_{0}=2$, for different values of the crystal momentum $\boldsymbol{q}$ in the first Brillouin zone: (a) $\left(q_{x}, q_{y}\right)=(0,0), \quad$ (b) $\left(q_{x}, q_{y}\right)=(\pi / a, 0), \quad$ (c) $\left(q_{x}, q_{y}\right)=(0, \pi / a),(\mathrm{d}) \quad\left(q_{x}, q_{y}\right)=(\pi / a, \pi / a)$ 
Figure 2: Magnetic energy bands $n=0,1$, of the square lattice as a function of the crystal momentum $\left(q_{x}, q_{y}\right)$ for (A) $\Phi / \Phi_{0}=2$. and (B) for $\Phi / \Phi_{0}=5 / 4$. The modulation strengths are $V_{x}=V_{y}=0.5 \mathrm{meV}$ and the energy eigenvalues are plotted in units of $\epsilon_{0}=\hbar \omega$. 


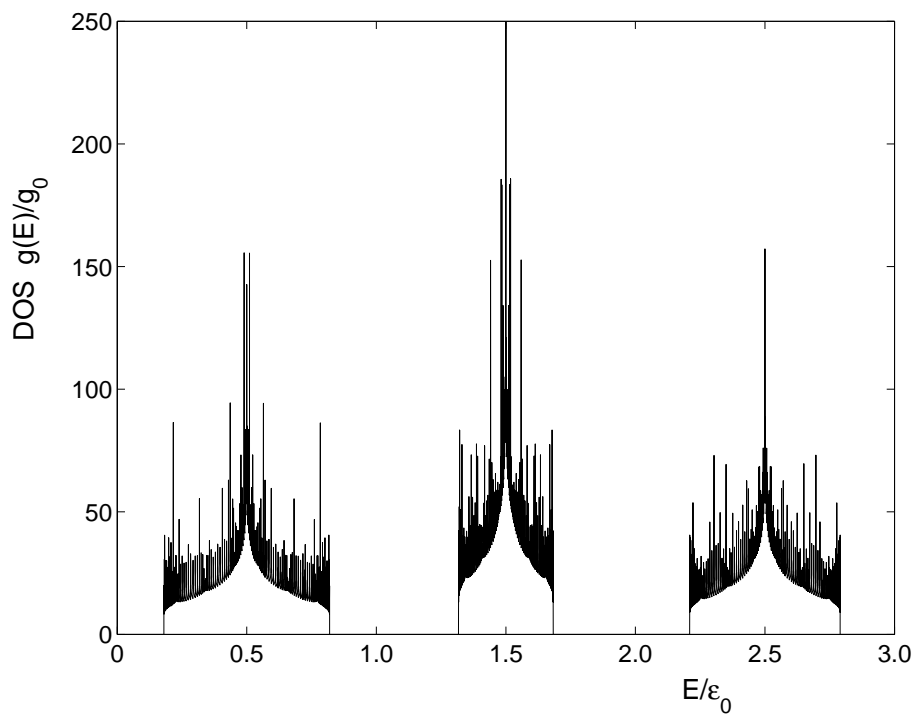

Figure 3: Density of states per unit area of the square lattice for the same values of parameters as in Fig.2. 
Figure 4: Magnetic energy bands $n=0,1$, of the triangular lattice as a function of the crystal momentum $\left(q_{x}, q_{y}\right)$ for or (A) $\Phi / \Phi_{0}=2$. and (B) for $\Phi / \Phi_{0}=5 / 4$. The modulation strengths are $V_{x}=V_{y}=0.5 \mathrm{meV}$ and the energy eigenvalues are plotted in units of $\epsilon_{0}=\hbar \omega$. 


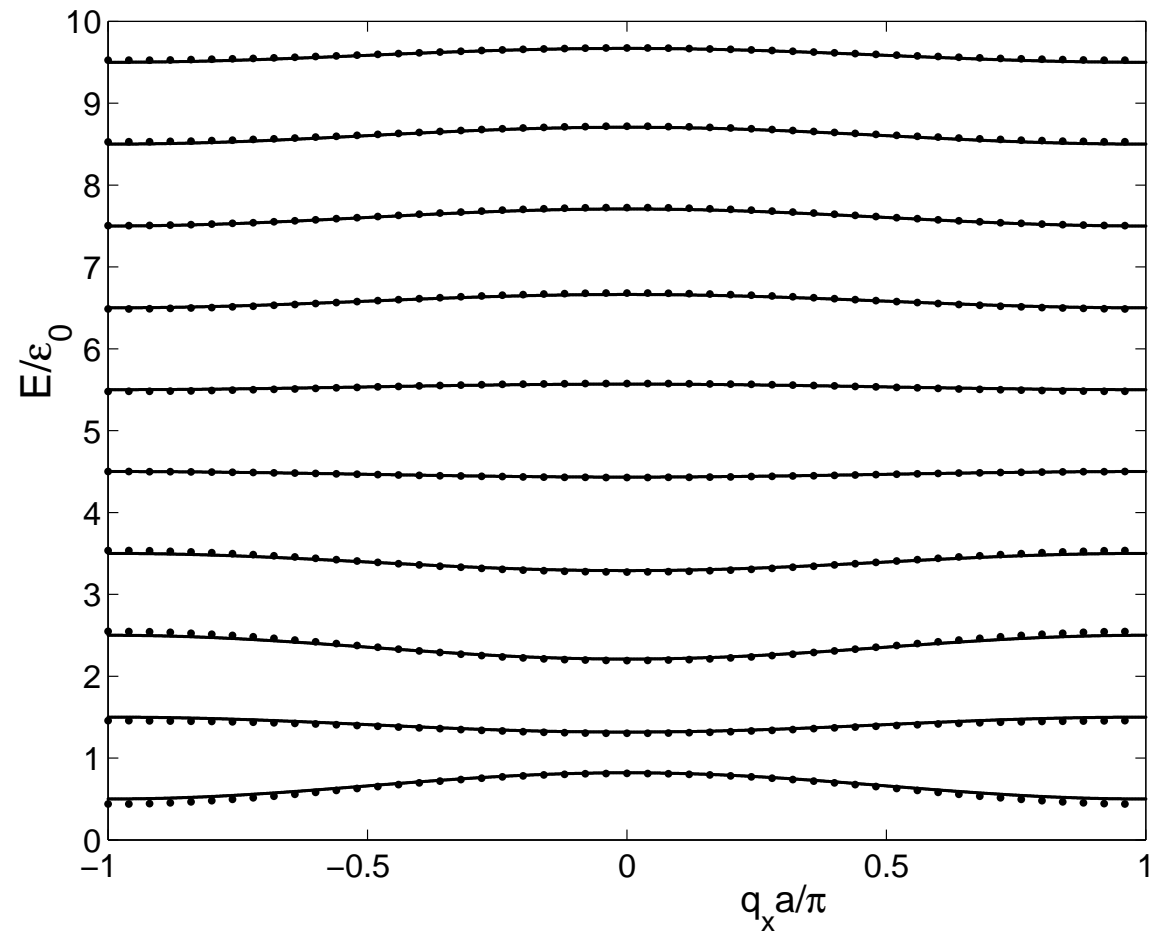

Figure 5: Energy spectrum of the square lattice as a function of the crystal momentum $\left(q_{x}, q_{y}=0\right)$ for the same values of parameters as in Fig.2. Solid lines are for the first order energy perturbation and dotted lines are for the exact energy spectrum. Only the lowest ten levels are shown. The energy eigenvalues are plotted in units of $\epsilon_{0}=\hbar \omega$ 
This figure "fig2a.jpg" is available in "jpg" format from: http://arxiv.org/ps/cond-mat/0609662v1 
This figure "fig2b.jpg" is available in "jpg" format from: http://arxiv.org/ps/cond-mat/0609662v1 
This figure "fig4a.jpg" is available in "jpg" format from: http://arxiv.org/ps/cond-mat/0609662v1 
This figure "fig $4 \mathrm{~b} . j p g$ " is available in "jpg" format from: http://arxiv.org/ps/cond-mat/0609662v1 